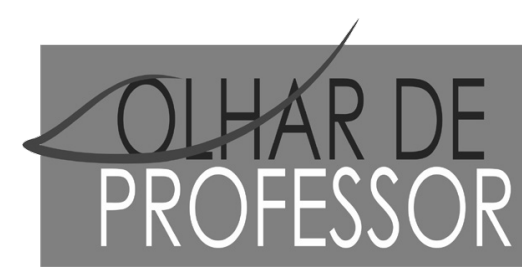

DOI: 10.5212/OLHARPROFR.v.2112.0011

\title{
A IMPORTÂNCIA DOS ESTÁGIOS SUPERVISIONADOS NO DESENVOLVIMENTO DO CONHECIMENTO PEDAGÓGICO DO CONTEÚDO
}

\author{
THE IMPORTANCE OF SUPERVISED INTERNSHIPS IN THE DEVELOPMENT OF PEDAGOGICAL CONTENT \\ KNOWLEDGE
}

\author{
LA IMPORTANCIA DE LASPRÁCTICAS SUPERVISADAS \\ ENELDESARROLLODELCONOCIMIENTODIDÁCTICODELCONTENIDO
}

\author{
LUCIANE FERNANDES DE GOES \\ KEYSY SOLANGE COSTA NOGUEIRA ${ }^{\text {** }}$ \\ CARMEN FERNANDEZ $Z^{\text {t*x }}$
}

\begin{abstract}
Resumo: O objetivo deste estudo foi investigar o Conhecimento Pedagógico do Conteúdo (PCK) de licenciandos de química durante a disciplina de Estágio Curricular Supervisionado (ECS), na qual o discente participa de um processo completo de ensino: planejamento, implementação e reflexão sobre a prática, sobre o assunto de reações redox. Para a análise dos dados, utilizou-se uma rubrica com elementos que representam o conteúdo e as estratégias instrucionais nos três momentos distintos. Cada elemento foi classificado em limitado, básico, proficiente ou exemplar. Dois pesquisadores analisaram nove relatórios finais da disciplina $(\mathrm{k}=0,92)$. Os resultados indicam que apesar dos futuros professores apresentaram desempenho limitado nos componentes do PCK, os ECS são espaços que fornecem elementos para a construção do PCK. Além disso, aponta-se para a necessidade de programas formação continuada, que forneçam subsídios para uma maturidade do conhecimento adquirido na formação inicial.
\end{abstract}

Palavras-chave: Conhecimento pedagógico do conteúdo (PCK). Estágio supervisionado. Reações redox.

\begin{abstract}
This study aimed to investigate the Pedagogical Content Knowledge (PCK) of chemistry undergraduates during the Supervised Curricular Internship (ECS), in which the student participates in a complete teaching process: planning, implementation and reflection on the practice, on the subject of redox reactions. For the the data analysis, a rubric with elements that represent the content and the instructional strategies in the three different moments was used. Each element was classified as limited, basic, proficient or exemplary. Two researchers analyzed nine final reports of the discipline $(\mathrm{k}=0.92)$. Although the future teachers have presented limited performance in the components of the PCK, the results indicate that the ECS are spaces that provide elements for the construction of the PCK. In addition, there is a need for continuing training programs that provide support for a maturity of knowledge acquired in initial training.
\end{abstract}

Keywords: Pedagogical content knoledge (PCK). Supervised internship. Redox reactions.

Resumen: El objetivo de este estudio fue investigar las manifestaciones el Conocimiento Didáctico del Contenido (PCK) de los futures profesores de química acerca de tema de reacciones redox, durante el Estadio curricular

\footnotetext{
* Doutora em Ensino de Ciências pela Universidade de São Paulo - USP-SP. Professora de educação básica no estado de São Paulo. E-mail: luciane.goes@usp.br

** Doutora em Ensino de Ciências pela Universidade de São Paulo - USP-SP. Professora da Universidade Federal de Santa Catarina. E-mail: keysy.nogueira@ufsc.br

*** Professora Livre Docente do Instituto de Química da Universidade de São Paulo - USP-SP. E-mail: carmen@iq.usp.br
} 
supervisado (PCS), la cual permite al future docente participar en un proceso de enseñanza completo que incluye la planificación de la lección, enseñanza y reflexión sobre su práctica. Para el análisis de datos, utilizamos una rúbrica con elementos que representan el contenido y las estrategias de instrucción en los tres momentos diferentes. Cada elemento ha sido clasificado como limitado, básico, competente o ejemplar. Para ello, dos investigadores analizaron nueve informes finales de la disciplina PCS $(\mathrm{k}=0,92)$. Los resultados indican que, si bien los futuros profesores presentaron un rendimiento limitado en los componentes de PCK, las PCS son ricos espacios que proporcionan elementos para la construcción de PCK. Además, señala la necesidad de programas de educación continua que proporcionen subsidios para una madurez del conocimiento adquirido en la capacitación inicial.

Palabras claves: Conocimiento didáctico del contenido (PCK). Estadio supervisado. Reacciones redox.

\section{INTRODUÇ̃̃̃O}

Nas últimas décadas diversos pesquisadores buscaram desvelar os conhecimentos que caracterizam a docência como profissão (TARDIF, 2010; GAUTHIER et al., 1998; SHULMAN, 1987). Shulman (1986, 1987) introduziu uma nova forma de pensar o conhecimento de professores, por meio da proposição de sete categorias para o conhecimento base: i.) conhecimento do conteúdo específico; ii.) conhecimento de contextos educativos; iii.) conhecimento de valores educativos e de seus objetivos; iv.) conhecimento pedagógico geral; v.) conhecimento curricular; vi.) conhecimento pedagógico do conteúdo (PCK) e; vii.) conhecimento das características, da cognição, entre outras, dos discentes.

Dentre os conhecimentos base, o PCK ganha destaque por representar o conhecimento que distinguiria o professor de um especialista no assunto (CHAN; HUME, 2019; FERNANDEZ, 2014; KIND, 2009; SHULMAN, 1987). O PCK seria a “[...] compreensão do que torna o aprendizado de tópicos fáceis ou difíceis: as concepções e preconceitos que estudantes de diferentes idades e contextos trazem consigo a aprendizagem dos tópicos e lições mais frequentemente ensinados.” (SHULMAN, 1986, p. 9, tradução nossa). Na percepção do autor, existe um corpo de conhecimentos que caracteriza o trabalho docente como profissão, pois "chamar algo de profissão é assumir que há uma base de conhecimentos amplamente construídos na academia.” (SHULMAN, 2004, p. 13). Pode-se dizer que o PCK inclui todo o conhecimento que um professor precisa para ensinar um assunto de forma eficaz. A aquisição do PCK é, portanto, uma tarefa central para aprender a ensinar. (SHULMAN, 1987; MAGNUSSON; KRAJCIK; BORKO, 1999). Acredita-se que este conhecimento pode ser muito útil para os professores em serviço e futuros professores (FERNANDEZ, 2014, 2015) para que durante suas formações guiadas por professores experientes, possam ampliar o repertório pedagógico. Por outro lado, alguns autores apontam para a perspectiva de que é possível e desejável desenvolver o PCK na formação inicial de professores. (VAN DRIEL; DE JONG, 2001).

Na literatura não há um consenso quanto ao conceito de PCK e, diversos autores propuseram modelos para defini-lo. (CHAN; HUME, 2019; GOES, 2014). Um dos modelos existentes na literatura, o modelo pentagonal, foi proposto por Park e Oliver (2008). Nesse modelo, os autores conceituam o PCK por meio dos cinco componentes: (i) orientações para o ensino de ciências, (ii) conhecimento do currículo de ciências, (iii) conhecimento do entendimento dos alunos em ciências, (iv) conhecimento de estratégias instrucionais para o ensino de ciências e (v) conhecimento de avaliação para o ensino de ciências. O modelo pentagonal, representado na figura 1, emergiu de um estudo de revisão bibliográfica dos trabalhos de Grossman (1990), Tamir (1988) e Magnusson, Krajcik e Borko (1999). (PARK; CHEN, 2012). Embora Magnusson, Krajcik e Borko (1999) tenham reconhecido a interação entre os cinco componentes, estruturaram o seu modelo de forma linear. Em contrapartida, o modelo pentagonal enfatiza essa inter-relação entre os componentes. Para os autores, o desenvolvimento do PCK consiste na integração de todos os componentes aliados à constante reflexão sobre a ação e a ação. 
Figura 1 - Modelo pentagonal do Conhecimento Pedagógico de Conteúdo para o Ensino de Ciências (PARK; OLIVER, 2008)

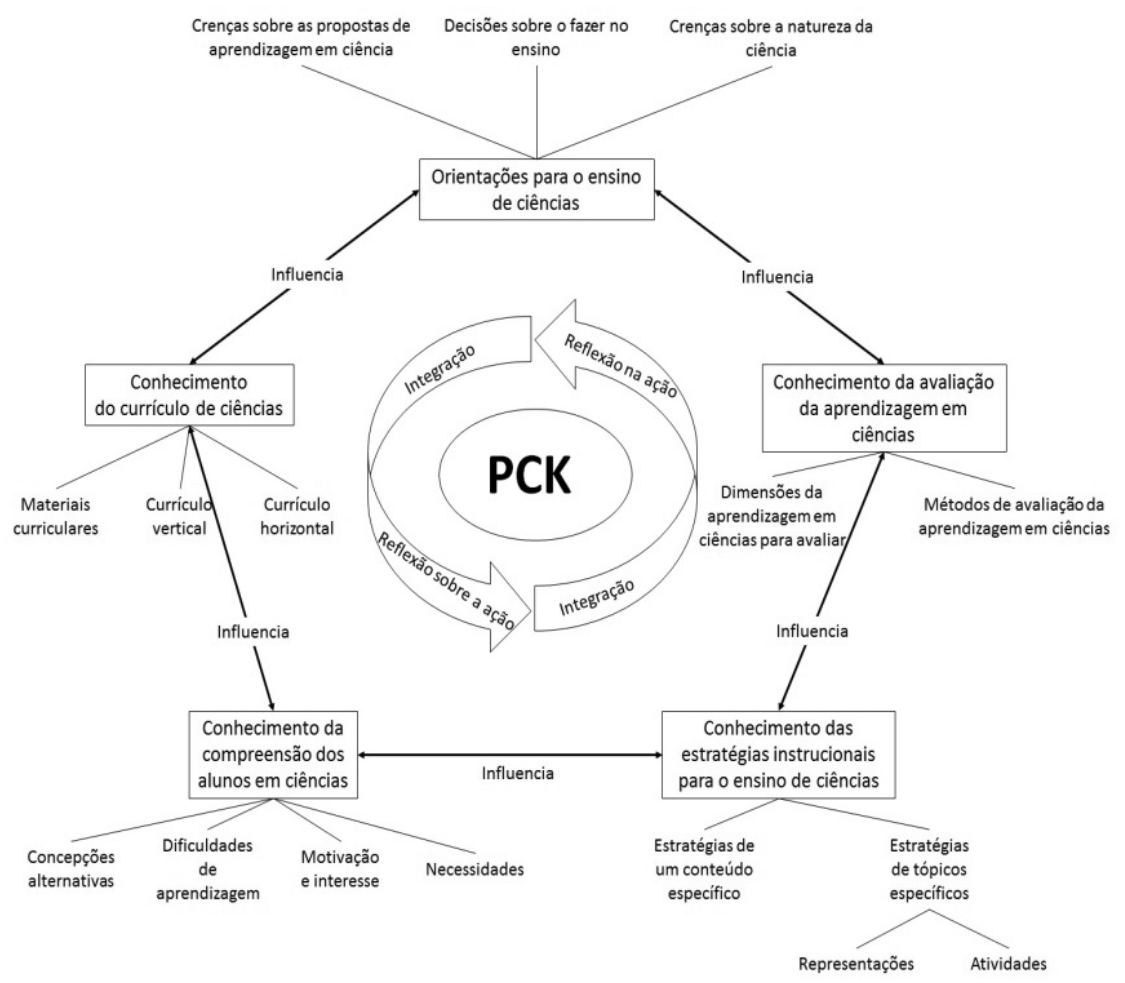

Fonte: FERNANDEZ, 2011.

No contexto brasileiro, um espaço no currículo de formação de professores que permitiria o desenvolvimento do PCK seria na disciplina de Estágio Curricular Supervisionado (ECS). (SILVA; SCHNETZLER, 2008). Para Almeida e Pimenta (2014) essa etapa que constitui a formação inicial de professores pode

[...] favorecer a mediação entre professores e alunos em formação no contexto real do exercício profissional através de estudos, análise, problematização, reflexão e proposição de soluções para o ensinar e o aprender, o compreender a reflexão sobre as práticas pedagógicas, trabalho docente e as práticas institucionais, situados em contextos sociais, históricos e culturais. (ALMEIDA; PIMENTA, 2014, p. 30).

Esse componente integrador no currículo das licenciaturas oportuniza ao licenciando desenvolver o trabalho pedagógico, promover a integração entre os conteúdos químicos e os pedagógicos, desenvolver o domínio da gestão de ensino, do planejamento das aulas, entre outros. As atividades no ECS ocorrem sob a orientação de um professor dos cursos de licenciatura e supervisão de um docente da educação básica. No ECS geralmente os futuros professores, estudam as condições determinantes da aprendizagem, do ensino e da produção de conhecimento em ambiente escolar, compreendam o contexto escolar, propõe, avaliam e adequam os materiais didáticos de acordo com os objetivos educacionais.

Por meio dos relatórios de estágio, os graduandos escrevem sobre suas propostas, intervenções e reflexões. Nessa perspectiva, para Freire (2002, p. 43-44) “[...] na formação permanente dos professores, o momento fundamental é o da reflexão crítica sobre a prática. É pensando criticamente a prática de hoje ou de ontem que se pode melhorar a próxima prática.” Considerando a vivência de futuros professores por meio de práticas didáticas e da reflexão sobre suas ações pedagógicas, pode-se considerar o ECS como um espaço de construção dos conhecimentos docentes, em virtude de oportunizar aos futuros professores à prática profissional.

Dessa forma, o objetivo deste estudo foi investigar as manifestações do PCK dos futuros professores de química durante o ECS, sobre o tema de reações redox. A reação de oxirredução foi escolhida como tema, porque é compreendida como um dos tópicos mais difíceis, para aprender e ensinar nas aulas 
de química. (DE JONG; TREAGUST, 2002; NOGUEIRA; GOES; FERNANDEZ, 2017). Além disso, são escassos os trabalhos que investigam o PCK na formação inicial de professor e consequentemente, como o conteúdo de reação de oxirredução vem sendo desenvolvido em sala de aula, pois geralmente investigam-se as limitações conceituais dos professores com este conteúdo. (ROLLNICK; MAVHUNGA, 2014; YILMAZ; BAYRAKÇEKEN, 2015).

Deste modo, esse fato justifica ainda mais o interesse em pesquisar como futuros professores de química trabalham este conteúdo em sala de aula, no contexto da disciplina de ECS. Em consonância, pretende-se responder a questão: será que na disciplina de ECS o licenciando mobiliza os conhecimentos de conteúdo específico e pedagógico, possivelmente construídos ao longo da licenciatura, ou seja, o PCK?

\section{ASPECTOS METODOLÓGICOS}

\section{NATUREZA DA PESQUISA}

Esta pesquisa é predominantemente qualitativa e figura se como estudo de caso, pois em um contexto específico buscou-se identificar a construção do PCK na disciplina de ECS. Na literatura, o estudo de caso compreende a compreensão de uma situação-problema, assim: “[...] o estudo de caso permite que uma investigação retenha as características holísticas e significativas dos eventos da vida real, como ciclos de vida individuais [...]”. (YIN, 2014, p. 3).

\section{CONTEXTO DE PESQUISA}

Este estudo foi realizado no curso de licenciatura em química do Instituto de Química da USP-SP. A Universidade de São Paulo foi criada em 1934 e dentro dela nasceu o Instituto de Química (IQ), que tinha sua equipe composta principalmente por cientistas alemães. A primeira aula de química foi formada, principalmente por médicos, dentistas, entre outros. Após três anos, o aluno era licenciado, mas esse título na época tinha outro significado, já que "licenciado" significava que o indivíduo possuía uma licença cultural ou científica. (MESQUITA; SOARES, 2011; SCHNETZLER, 2002).

Foi somente em 1962 que foi estabelecido um currículo mínimo específico para licenciatura em química, na Faculdade de Filosofia, Ciências e Letras da Universidade de São Paulo (FFCL-USP). Posteriormente, a licenciatura em Química foi incorporada ao QI e somente em 1993, com o início das atividades do curso noturno de Química, que o curso teve seu currículo modificado e as disciplinas pedagógicas começaram a ser desenvolvidas ainda no terceiro período. (SCHNETZLER, 2002).

O curso de Licenciatura em Química (2017) era estruturado em 8 semestres. Uma das fases fundamentais contempladas em seu currículo são as disciplinas de ECS, com 400 horas. Na licenciatura, os futuros professores de química são inseridos na escola, por meio do ECS, para que possam conhecer as instituições de ensino da educação básica, desenvolver e implementar propostas de ensino, entre outras.

\section{PARTICIPANTES}

Participaram deste estudo nove licenciandos em química da Universidade de São Paulo. Os futuros professores de química estavam cursando a disciplina de ECS. Todos licenciandos desenvolveram e implementaram aulas sobre o conteúdo de reações redox. Por questões de confidencialidade, todos os participantes receberam pseudônimos. Na Tabela 1 estão disponíveis as características gerais dos participantes desta pesquisa. 
Tabela 1 - Características dos participantes

\begin{tabular}{c|c|c}
\hline Participantes & Tempo de experiência & Período no curso de Licenciatura \\
\hline FP1 & 5 anos & $6^{\text {o semestre }}$ \\
\hline FP2 & 3 anos & $8^{\text {o semestre }}$ \\
\hline FP3 & 1 ano & $8^{\text {o } \text { semestre }}$ \\
\hline FP4 & menos de 1 ano & $6^{\text {o semestre }}$ \\
\hline FP5 & 1 ano & $6^{\text {o semestre }}$ \\
\hline FP6 & 5 anos & $6^{\text {o semestre }}$ \\
\hline FP7 & 2 anos & $8^{\text {o semestre }}$ \\
\hline FP8 & 2 anos & $8^{\text {o semestre }}$ \\
\hline FP9 & sem experiência & $8^{\text {o semestre }}$ \\
\hline
\end{tabular}

Fonte: os autores.

\section{COLETA DE DADOS}

A coleta de dados foi realizada por meio dos relatórios finais produzidos pelos licenciandos em química na disciplina de ECS. Ao longo da referida disciplina, os alunos foram orientados pela professora responsável do curso, a produzir um relatório final que contemplasse o plano de ensino, a implementação da intervenção e uma reflexão sobre esse processo. O relatório tinha como estrutura base a análise do Projeto Político Pedagógico (PPP) da escola da educação básica, na qual os licenciandos desenvolveram o ECS, era estruturado ainda, pelos planejamentos de ensino, pelas observações realizadas ao longo do estágio, entre outros. Além disso, os licenciandos em química descreveram o plano de ensino, a série para qual ministraram suas aulas, os conteúdos químicos, a duração das aulas, as estratégias de ensino adotadas, a descrição da intervenção de ensino, a sua análise e, por fim, descreveram como a disciplina de ECS contribuiu para a sua formação como futuro professor.

\section{ANÁLISE DE DADOS}

Os resultados foram analisados por meio das rubricas propostas por Park et al. (2011) para investigar dois componentes do PCK: conhecimento do entendimento do aluno com respeito a um determinado assunto (KSU) e conhecimento de estratégias instrucionais e representações do assunto (KISR). As rubricas consistem em nove elementos independentes para representar o KSU e o KISR em três momentos diferentes: planejamento (P), implementação (I) e reflexão (R).

Na fase de planejamento existem 4 elementos: Entendimento do conhecimento prévio (UPK), Estratégias instrucionais para adaptar o conhecimento prévio (ISPK), Entendimento das Dificuldades dos alunos (LD), Estratégias instrucionais para adaptar as dificuldades de aprendizagem (ISLD). Na fase de implementação existem 3 elementos: Questões para investigar a compreensão do aluno (QSU), Espontaneidade para desafiar equívocos ou resolver dificuldades de aprendizagem (SMLD), Justificativa para estratégias e representações instrucionais (RISR). E na fase de reflexão existem 2 elementos: Foco na compreensão do aluno (SU), Alteração das estratégias e representações instrucionais (IS).

Cada elemento foi classificado de acordo com o nível de performance, usando uma escala de quatro pontos: 1 = "Limitado", 2 = "Básico", 3 = "Proficiente" e 4 = "Exemplar", totalizando 36 pontos possíveis para o instrumento como um todo.

Os relatórios dos licenciandos em química foram analisados de forma independente, por dois avaliadores, doutores em Ensino de Ciências. A concordância entre os avaliadores foi feita por meio do índice de Kappa, que corresponde a um procedimento estatístico que revela o número de respostas concordantes entre os interobservadores. Esta medida tem valor máximo de 1, o que indicaria uma concordância perfeita; valores próximos de 0 indicam que não há concordância entre os avaliadores. Segundo Landis e Koch (1977), os valores de Kappa que classificam os diferentes níveis de concordância podem indicar as interpretações apresentadas na tabela 2. 
Tabela 2 - Classificação dos níveis de concordância em relação ao valor do Kappa (LANDIS; KOCH, 1977, tradução MATOS, 2014)

\begin{tabular}{c|c}
\hline Valor do coeficiente Kappa & Nível de concordância \\
\hline $\mathrm{k}<0$ & Sem concordância \\
\hline $0 \leq \mathrm{k}<0,21$ & presença de ligeira concordância \\
\hline $0,21 \leq \mathrm{k}<0,41$ & concordância fraca \\
\hline $0,41 \leq \mathrm{k}<0,61$ & concordância moderada \\
\hline $0,61 \leq \mathrm{k}<0,81$ & concordância excelente \\
\hline $0,81 \leq \mathrm{k} \leq 1,0$ & concordância quase perfeita \\
\hline
\end{tabular}

De modo geral, pode-se compreender que a concordância será considerada satisfatória quando o Kappa tiver valores superiores a 0,60. (LANDIS; KOCH, 1977).

\section{RESULTADOS E DISCUSSÃO}

A análise dos relatórios produzidos pelos licenciandos em química, possibilitaram identificar a manifestação de dois componentes do PCK no contexto da disciplina de ECS, a saber: Conhecimento do entendimento do aluno com respeito a um determinado assunto (KSU) e conhecimento de estratégias instrucionais e representações do assunto (KISR). Neste sentido, na tabela 3 apresenta-se os resultados da análise por meio das rubricas de Park et al. (2011).

Tabela 3 - Resultado dos componentes KSU e KIRS dos futuros professores de química (Lim=limitado; Bás=Básico; Prof=Proficiente; FP=Futuro Professor)

\begin{tabular}{|c|c|c|c|c|c|c|c|c|c|}
\hline \multirow{2}{*}{ Participantes } & \multicolumn{4}{|c|}{ Planejamento } & \multicolumn{3}{|c|}{ Implem entação } & \multicolumn{2}{|c|}{ Reflexão } \\
\hline & P-UPK & P-ISPK & P-LD & P-ISLD & I-QSU & I-SMLD & I-RISR & R-SU & R-IS \\
\hline FP - 1 & $\operatorname{Lim}$ & $\operatorname{Lim}$ & $\operatorname{Lim}$ & $\operatorname{Lim}$ & Bás & $\operatorname{Lim}$ & $\operatorname{Lim}$ & $\operatorname{Lim}$ & Bás \\
\hline FP - 2 & Bás & Bás & Bás & $\operatorname{Lim}$ & Bás & Bás & Bás & Prof & Bás \\
\hline FP - 3 & $\operatorname{Lim}$ & $\operatorname{Lim}$ & $\mathrm{Lim}$ & $\operatorname{Lim}$ & Bás & Bás & Bás & $\operatorname{Lim}$ & $\operatorname{Lim}$ \\
\hline FP - 4 & $\operatorname{Lim}$ & $\operatorname{Lim}$ & $\operatorname{Lim}$ & $\operatorname{Lim}$ & Bás & $\operatorname{Lim}$ & $\operatorname{Lim}$ & Bás & $\operatorname{Lim}$ \\
\hline FP - 5 & $\mathrm{Lim}$ & $\mathrm{Lim}$ & $\operatorname{Lim}$ & $\mathrm{Lim}$ & Bás & $\operatorname{Lim}$ & $\operatorname{Lim}$ & $\operatorname{Lim}$ & $\mathrm{Lim}$ \\
\hline FP - 6 & $\mathrm{Lim}$ & $\operatorname{Lim}$ & $\operatorname{Lim}$ & $\operatorname{Lim}$ & $\operatorname{Lim}$ & $\operatorname{Lim}$ & $\operatorname{Lim}$ & $\operatorname{Lim}$ & $\mathrm{Lim}$ \\
\hline FP - 7 & $\operatorname{Lim}$ & $\operatorname{Lim}$ & $\operatorname{Lim}$ & $\operatorname{Lim}$ & Bás & Bás & Bás & $\operatorname{Lim}$ & $\mathrm{Lim}$ \\
\hline FP - 8 & Bás & $\operatorname{Lim}$ & Bás & $\operatorname{Lim}$ & Bás & Bás & $\operatorname{Lim}$ & Bás & $\operatorname{Lim}$ \\
\hline FP - 9 & Bás & $\operatorname{Lim}$ & Bás & Bás & Bás & Bás & Bás & Prof & Bás \\
\hline
\end{tabular}

O valor do Kappa encontrado foi de 0,92, o que indicou concordância excelente na análise dos dois avaliadores,com relação ao PCK dos futuros professores de química.

De acordo com os dados, observa-se que nenhum dos futuros professores possui componentes classificados como exemplares. Grande parte dos licenciandos tem os componentes KSU e KIRS classificados como limitados, porque geralmente não levaram em conta o conhecimento prévio dos alunos sobre as reações redox, não integraram o conhecimento prévio com as estratégias instrucionais e não buscaram considerar a compreensão do aluno para adotar novas estratégias de ensino.

Ao se esmiuçar separadamente as diferentes etapas que constituíam o relatório, observa-se que, na fase de planejamento, $77 \%$ dos elementos foram classificadas em limitado. Na fase de implementação, a situação difere um pouco, pois a maioria dos licenciandos tiveram os elementos classificados em básico (63\%). No estágio de reflexão, a maioria dos elementos, ainda são classificados como limitados (61\%), mas a diferença observada nessa fase é que dois licenciandos foram classificados como proficientes (11\%). Este resultado, de certa forma, difere do que se esperava, pois, os participantes deste estudo, por ainda serem licenciandos e estarem em contato com discussões teóricas sobre o processo de ensino-aprendizado, acreditava-seque teriam um melhor desempenho na etapa de planejamento, por ser uma etapa que mobilizaria um conhecimento teórico. 
A análise dos dois componentes investigados do PCK (conhecimento da compreensão do aluno KSU e conhecimento de estratégias e representações instrucionais - KISR), os denotam que o KSU tem um melhor nível de desempenho, sendo 50\% dos licenciandos classificados como básico, do que o KISR, em que $69 \%$ foram classificados como limitados.

Na tabela 3, apresentada anteriormente, pode-se observar que na categoria planejamento (P), para a subcategoria P-LD (Dificuldade dos alunos), apenas três participantes demonstram algum conhecimento sobre as dificuldades dos alunos relacionadas às reações redox:

Percebi, observando as aulas iniciais, que os alunos sabiam como equilibrar uma equação eletroquímica, mas memorizaram um algoritmo para resolver os exercícios. Ficou claro que os alunos sabiam como resolver problemas muito bem, mas não poderíamos atribuir nenhum significado fenomenológico à equação. (FP-9, P-LD, básico).

Na subcategoria P-ISPK, apenas um dos participantes integra de forma considerada básica o conhecimento prévio dos discentes, incluindo os equívocos conceituais. Os demais participantes foram classificados como limitados. Por exemplo, ao se analisar um trecho do relatório do licenciando 8 na sua descrição dos objetivos da aula, observa-se que o mesmo não considera as dificuldades dos alunos em relação a esse conteúdo, em nenhum momento de seu planejamento, pois o discente descreveu que "O primeiro experimento foi sobre a oxidação, porque os estudantes tinham acabado de ver esse assunto.” (FP-8, P-ISPK, limitado).

Nesse exemplo, nota-se que a justificativa do licenciando para a adoção do experimento foi fundamentada apenas no sequenciamento do conteúdo, o licenciando não justifica, por exemplo, como o experimento poderia contribuir para a compreensão dos alunos sobre reações redox.

Em relação a compreensão do conhecimento prévio (UPK) na fase de planejamento, três licenciandos apresentaram empenho considerado básico do conhecimento comum prévio dos discentes da educação básica.Nesse sentido, FP-8 buscou considerar as limitações associadas a reações redox descritas na literatura em seu planejamento, como sugere o trecho:

O conceito de óxido-redução geralmente não é de fácil entendimento para os alunos e na literatura encontram-se muitos trabalhos que reportam estas dificuldades a respeito do processo de ensino aprendizagem vinculadas a este tema. (FP-8, P-UPK, básico).

De modo semelhante as autoras Santos e Freire (2017) ao pesquisarem o desenvolvimento do PCK de licenciandos em química, no contexto da disciplina de ECS, identificaram que duas licenciandas buscaram levar em consideração as dificuldades dos discentes em seus planejamentos, sobre o conteúdo de cinética química.

Na subcategoria P-ISLD (Estratégias instrucionais para acomodar a dificuldade de aprendizagem) oitos dos nove licenciandos em química não integraram as dificuldades de aprendizagem dos discentes, que são comuns no ensino de reações redox, nas estratégias instrucionais adotadas em seus planejamentos. Apenas um licenciando teve um desempenho considerado básico:

Uma revisão teórica dos conceitos da pilha de Daniel foi feita no início da aula com o objetivo de esclarecer algumas dúvidas [...] e evitar algumas concepções alternativas já relatadas na literatura. (FP-9, P-ISLD, básico).

Nesse exemplo, observa-se que a escolha da estratégia, revisão de um conceito prévio, foi devido o reconhecimento das dificuldades dos alunos e do conhecimento referente às concepções de alunos sobre o conteúdo de reações redox relatadas na literatura.

Na fase de implementação (I), para a subcategoria I-QSU (questões para investigar a compreensão do aluno), a maioria dos participantes relatam que utilizam questões para examinar a compreensão ou não dos alunos:

Enquanto os alunos estavam preparando o experimento, eu andei pelo laboratório e perguntei por que eles estavam fazendo isso. [...] eu fiz essas perguntas para instigar os alunos e investigar seus entendimentos. (FP-7, I-QSU, básico). 
De acordo ainda, com os resultados dispostos na tabela 3, entre os nove futuros professores, apenas dois deles apresentaram um elemento categorizado como proficiente, e esse desempenho foi observado na fase de reflexão. A seguir, apresenta-se um trecho do relatório do futuro FP- 9, que tornou possível avaliar o R-SU (Foco na compreensão do aluno) como proficiente:

[...] os alunos foram solicitados a calcular o potencial padrão [...] muitos alunos não puderam responder [...] porque não conseguiram escrever as reações de redução e oxidação. Observa-se que os alunos não entenderam o fenômeno. (FP-9, R-SU, proficiente).

Esses mesmos dois licenciandos (FP-2 e FP-9) apresentam a maioria dos elementos do PCK categorizados como básicos. Apresenta-se abaixo um dos trechos do relatório classificado na P-LD como básica:

Percebi, observando as aulas iniciais, que os alunos sabiam como equilibrar uma equação eletroquímica, mas memorizaram um algoritmo para a resolução de exercícios. Ficou claro que os alunos sabiam como resolver problemas muito bem, mas não poderíamos atribuir nenhum significado fenomenológico à equação. (FP-2, P-LD, básico).

Ao analisar as características desses futuros professores de química individualmente, se observa alguns aspectos que podem influenciar no seu desempenho. O FP-2 era professor, então já lecionava antes de iniciar seu curso de licenciatura em química. O FP-9, apesar de não ter experiência em docência, durante o ECS, foi tutorado por uma professora da educação básica, com mais de 30 anos de experiência em sala de aula. Desta forma, em sua reflexão, o FP-9 relata a contribuição da professora supervisora durante sua vivência no ECS. Além disso, FP-9 discorreu sobre a importância de uma das disciplinas do Cursos de Licenciatura em Química, que influenciaram em sua regência:

Graças ao suporte teórico recebido através das aulas de Metodologia do Ensino de Química, puderam-se analisar quais eram os padrões de abordagem predominantes durante as falas da intervenção. O tipo de estratégia utilizada na exposição da intervenção era baseado em uma abordagem interativo-dialógica que se diferenciou das aulas de observação que se mantiveram numa abordagem de autoridade - não dialógica. (FP-9).

A narrativa do licenciando em química denota que a disciplina de ECS está propiciando aos futuros professores integrarem os conhecimentos aprendidos na licenciatura, com os vivenciados na escola da educação básica. (PIMENTA; LIMA, 2004).

Outro aspecto que emergiu na análise dos resultados, sugere que não houve uma relação entre o tempo de experiência e o desenvolvimento do PCK. Entretanto, o que se observa é uma correlação entre o momento dos licenciandos no curso e seus desempenhos. Nesse sentido, os licenciandos em química mais avançados no curso, ou seja, aqueles prestes a finalizarem a licenciatura em química, demonstraram um melhor desenvolvimento dos componentes do PCK em relação aos demais licenciandos. Em consonância, infere-se que o curso de licenciatura em química, de alguma maneira influencia e contribui para a prática docente e, consequentemente para o desenvolvimento do PCK.

\section{CONCLUSÕES}

Esse estudo reforça a ideia de que o ECS é um espaço rico de reflexão que possibilita o diálogo entre a teoria e prática, um processo importante na formação de professores. Os resultados indicam que os licenciandos em química têm um desempenho limitado para os dois componentes investigados do PCK (conhecimento dos estudantes e conhecimento das estratégias instrucionais), principalmente na fase de planejamento. Este fato aponta para a necessidade de desenvolver os componentes do PCK na formação inicial, a fim de permitir uma melhoria no planejamento das aulas e estimular a reflexão dos futuros professores sobre suas ações pedagógicas. Considerando que o estágio é a última fase da formação inicial do professor, espera-se que os professores em formação já apresentem um maior conhecimento tanto do conteúdo quanto das estratégias, principalmente sobre planejamento. Assim, as licenciaturas devem ser planejadas para possibilitar a expansão do PCK e integração de seus componentes. 
Os resultados sugerem que os relatórios finais podem ser um bom dado para a análise de componentes do PCK, pois no relatório há uma descrição do planejamento, das aulas implementadas e reflexões dos futuros professores sobre suas experiências iniciais em sala de aula. Com a adoção das rubricas, foi possível investigar dois componentes do PCK. A fim de investigar outros componentes do PCK, seria necessário usar outras metodologias de acesso, por exemplo, entrevistas ou observações gravadas em vídeo, bem como outra forma de análise de dados que englobe os outros componentes.

\section{AGRADECIMENTOS}

As autoras agradecem aos licenciandos em química, que participaram desta pesquisa e ao apoio financeiro da Fapesp, processos nº 2013/07937-8, nº 2014/14356-4 e n²016/08677-8.

\section{REFERÊNCIAS}

ALMEIDA, M. I.; PIMENTA, S. G. (orgs.). Estágios supervisionados na formação docente: educação básica e educação de jovens e adultos. São Paulo: Cortez, 2014.

CHAN, K. K. H.; HUME, A. Towards a consensus model: literature review of how science teachers' pedagogical content knowledge is investigated in empirical studies. In.: HUME, A.; COOPER, R.; BOROWSKI, A. (eds.). Repositioning Pedagogical Content Knowledge in teachers' knowledge for science teaching. Singapore: Springer, 2019. p. 1-49.

DE JONG, O. D. J.; TREAGUST, D. F. The teaching and learning of electrochemistry. In.: GILBERT, J. K.; DE JONG, O. D. J.; JUSTI, R.; TREAGUST, D. F.; VAN DRIEL, J. H. (eds.). Chemical education: towards research-based practice. Kluwer: Dordrecht, 2002. p. 317-337.

FERNANDEZ, C. Revisitando a base de conhecimentos e o conhecimento pedagógico do conteúdo (PCK) de professores de ciências. Revista Ensaio, Belo Horizonte, v. 17, n. 2, p. 500-528, maio/ago. 2015.

FERNANDEZ, C. Knowledge base for teaching and Pedagogical Content Knowledge (PCK): some useful models and implications for teachers' training. Problems of Education in the Twenty First Century, Siauliai, v. 60, s/n, p. 79-100, july 2014.

FERNANDEZ, C. PCK - Conhecimento Pedagógico do Conteúdo: perspectivas e possibilidades para a formação de professores. In.: ENCONTRO NACIONAL DE PESQUISA EM EDUCAÇÃO EM CIÊNCIAS, 8., 2011, Campinas. Atas [...] Rio de Janeiro: UFRJ, 2011.

FREIRE, P. Pedagogia da autonomia: saberes necessários à prática educativa. São Paulo: Paz e Terra, 2002.

GAUTHIER, C; MARTINEAU, S.; DESBIENS, J.; MALO, A.; SIMARD, D. Por uma teoria da pedagogia: pesquisas contemporâneas sobre o saber. Ijuí: Unijuí, 1998.

GOES, L. F. Conhecimento Pedagógico do Conteúdo: estado da arte no campo da educação e no ensino de Química. 2014, 155 f. Dissertação (Mestrado em Ensino de Ciências) - Instituto de Física; Instituto de Química; Instituto de Biociências; Faculdade de Educação, Universidade de São Paulo, São Paulo, 2014.

KIND, V. Pedagogical content knowledge in science education: perspectives and potential for progress. Studies in Science Education, London, v. 45, n. 2, p. 169-204, aug. 2009.

LANDIS, R. J.; KOCH, G. G. The measurement of observer agreement for categorical data. Biometrics, v. 33, n. 1, p. 159-174, mar. 1977.

MAGNUSSON, S.; KRAJCIK, L.; BORKO, H. Nature, sources and development of pedagogical content knowledge. In.: GESS-NEWSOME, J.; LEDERMAN, N. G. (eds.). Examining pedagogical content 
knowledge: the construct and its implications for science education. Dordrecht, The Netherlands: Kluwer Academics, 1999. p. 95-132.

MATOS, D. A. S. Confiabilidade e concordância entre juízes: aplicações na área educacional. Est. Aval. Educ., São Paulo, v. 25, n. 59, p. 298-324, set./dez. 2014.

MESQUITA, N. A. da S.; SOARES, M. H. F. B. Aspectos históricos dos cursos de licenciatura em Química no Brasil nas décadas de 1930 a 1980. Química Nova, São Paulo, v. 34, n. 1, p. 165-174, jan./ dez. 2011.

NOGUEIRA, K. S. C.; GOES, L. F.; FERNANDEZ, C. As limitações de ensino-aprendizagem associadas ao conteúdo redox nos eventos brasileiros. Ensenanza de las Ciencias, Sevilla, n. extra, p. 4197-4202, sep. 2017.

PARK, S.; JANG, J. Y.; CHEN, Y. C.; JUNG, J. Is Pedagogical Content Knowledge (PCK) necessary for reformed science teaching? Evidence from an empirical study. Research in Science Education, v. 41, n. 2, p. 245-260, dec. 2011.

PARK, S.; OLIVER, J. S. National Board Certification (NBC) as a catalyst for teachers' learning about teaching: the effects of the NBC processon candidate Teachers' PCK development. Journal of Research in Science Teaching, Hoboken, v. 45, n. 7, p. 812-834, sep. 2008.

PIMENTA, S. G.; LIMA, M. S. L. Estágio e docência. São Paulo: Cortez, 2004.

ROLLNICK, M.; MAVHUNGA, E. PCK of teaching electrochemistry in chemistry teachers: a case in Johannesburg, Gauteng Province, South Africa. Educación Química, Ciudad de México, v. 25, n. 3, p. 354-362, june 2014.

SANTOS, E. A.; FREIRE, L. I. F. Planejamento e aprendizagem docente durante o estágio curricular supervisionado. Actio, Curitiba, v. 2, n. 1, p. 263-281, jan./jul. 2017.

SCHNETZLER, R. P. A pesquisa em ensino de química no Brasil: conquistas e perspectivas. Química Nova, São Paulo, v. 25, supl. 1, p. 14-24, maio 2002.

SHULMAN. L. S. Professing the liberal arts. In.: SHULMAN, L.S. Teaching as community property. Essays on higher education. San Francisco: Jossey-Bass, 2004. p. 12-31.

SHULMAN, L. Knowledge and teaching: foundations of the new reform. Harvard Educational Review, Cambridge, v. 57, n. 1, p. 1-22, feb. 1987.

SHULMAN, L. Those who understand: knowledge growth in teaching. Educational Researcher, v. 15, n. 2, p. 4-14, feb. 1986.

SILVA, R. M. G.; SCHNETZLER, R. P. Concepções e ações de formadores de professores de Química sobre o estágio supervisionado: propostas brasileiras e portuguesas. Química Nova, São Paulo, v. 31, n. 8, p. 2174-2183, dez. 2008.

TAMIR, P. Subject matter and related pedagogical knowledge in teacher education. Teaching and Teacher Education, Washington DC, v. 4, n. 2, p. 99-110, apr. 1988.

TARDIF, M. Saberes docentes e formação profissional. Petrópolis: Vozes, 2010.

VAN DRIEL, J. H.; DE JONG, O. Investigating the development of preservice teachers' Pedagogical Content Knowledge. Paper presented at the Annual Meeting of the National Association for Research in Science Teaching, St Louis, Mo. 1-39, 2001.

YILMAZ, A.; BAYRAKÇEKEN, S. Determining of the prospective teachers' understandings of electrochemistry. Procedia - Social and Behavioral Sciences, v. 174, p. 2831-2838, feb. 2015.

YIN, R. K. Case study research design and methods. Thousand Oaks: Sage, 2014. 